\title{
Simulation-optimization Model for Design of Water Distribution System using Ant Based Algorithms
}

\author{
R. Moeini", a, and S.A.M. Moulaei ${ }^{b}$ \\ aDepartment of Civil Engineering, Faculty of Civil Engineering and Transportation, University Of Isfahan, 81746-73441, \\ Isfahan, Iran. \\ bBaft Imam Hussein Faculty of Engineering, Professional Technical University, 7851915848, Azadegan Street, \\ Baft, Kerman, Iran.
}

Received 28 January 2015; Accepted 7 February 2017

\begin{abstract}
In this paper, the Ant Colony Optimization Algorithm (ACOA) is applied to solve Water Distribution System design optimization problem proposing two different methods. Considering pipe diameters as decision variables of the problem, Ant System and Max-Min Ant System, referred to ACOA1 and ACOA2 respectively, are applied to determine pipe diameters. In proposed methods, the ant-based models are interfaced with EPANET as simulator for the hydraulic analysis. Three benchmark test examples are solved with proposed methods and the results are presented and compared with those obtained with other existing methods. The results show the capability of the proposed methods to optimally solve the design optimization problem in which best results are obtained with ACOA2 in comparison with other available results. Furthermore, the results show the superiority of the proposed ACOA2 over than the ACOA1 in which the trade-off between the two contradictory search characteristic of exploration and exploitation is managed better by using MaxMin Ant System.
\end{abstract}

Keywords: Water distribution system; Ant colony optimization algorithm; EPANET; Optimal design; Pipe diameter.

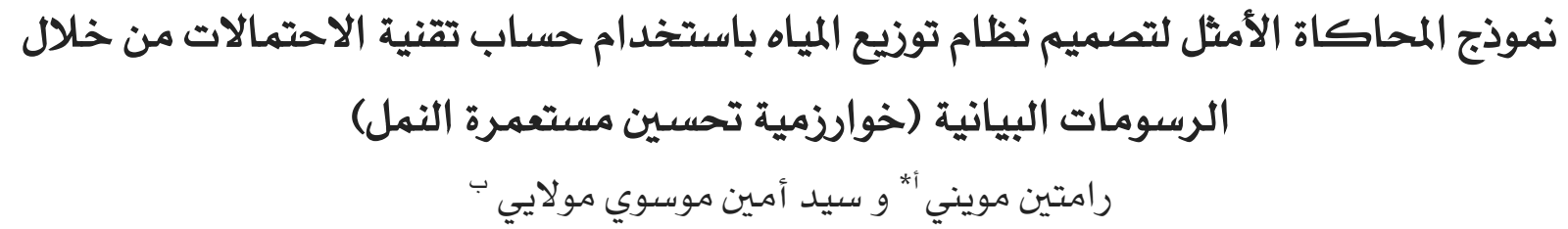

الملخص : قام هذا البحث بتطبيق حساب تقنية الاحتمالات من خلال الرسومات البيانية (أكوا) لحل مشكلة تحسين تصميم

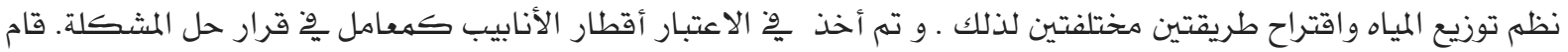

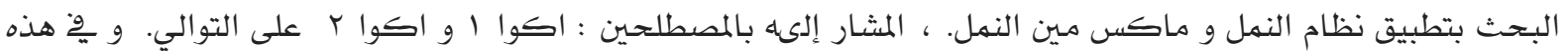

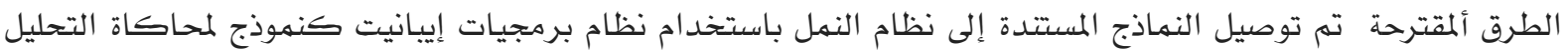

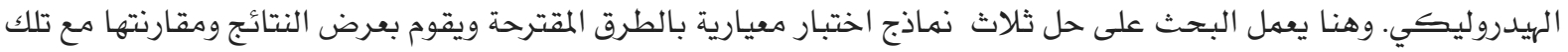

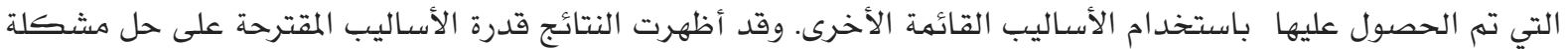

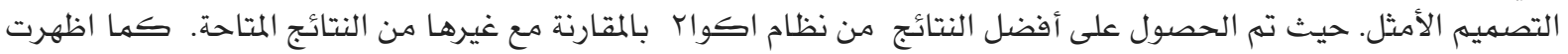

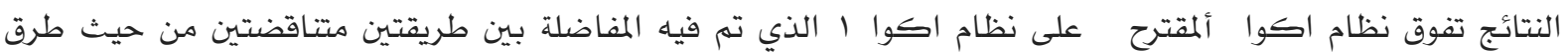

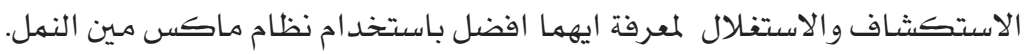

الكلمات المفتاحية : نظام توزيع المياه، خوارزمية تحسين مستعهرة النهل، نظام برهجيات إيبانيت،التصهيم الأمثل،قطر الأنابيب.

* Corresponding author's e-mail: r.moeini@eng.ui.ac.ir 


\section{Introduction}

A Water Distribution System (WDS) is a manmade system that is used to convey water from the source to the user. The main aim of every WDS is to deliver water to the user when it is necessary, in the correct quantity and in accordance with relevant quality standards. WDSs are therefore considered to be essential infrastructure in every modern community. As WDSs continue to get aged and cities continue to grow, the design of new WDSs and the rehabilitation or upgrading of existing WDSs will continue to be a central problem. Construction of WDS is very expensive due to cost of WDS components. A quite small change in the WDS components, therefore, leads to an enormous saving. The general WDS design optimization problem, therefore, involves minimizing the system cost subject to hydraulic constraints such as meeting minimum allowable pressure and/or maximum allowable velocity constraints under design demand levels.

A significant amount of research has focused on the optimal design, upgrade or rehabilitation of WDSs. In general, the available methods can be classified as 1) Linear programming (LP), 2) Nonlinear programming (NLP), 3) Dynamic programming (DP) and 4) Evolutionary Algorithm (EA) such as Genetic Algorithm (GA), Simulated Annealing (SA), Shuffled Frog Leaping Algorithm (SFLA), Tabu Search (TS), Ant Colony Optimization Algorithm (ACOA), Harmony Search (HS), Particle Swarm Optimization (PSO), Cross Entropy (CE), Honey-Bee Mating Optimization (HBMO) and Differential Evolution (DE) in which they were usually used for optimal design of WDS. Haghighi et al. (2011) listed different methods applied to design of WDS such as enumeration (Gessler 1985; LP (Bai et al. 2007; NLP Xu and Goulter 1999; DP Schaake and Lai 1969; GA Jian and Yanbing 2010; Bi et al. 2015; SA Costa et al. 2000; SFLA Baoyu et al. 2011; TS Lippai et al. 1999; ACOA Afshar 2007; HS Yang et al. 2012); PSO Babu and Vijayalakshmi 2013; HBMO Mohan and Babu 2010; CE Shibua and Janga Reddya 2012; DE Dong et al. 2012 and other EA and hybrid methods Zhou et al. 2016; Sheikholeslami et al. 2016) in which some of them are presented in this paper. Each of these optimization methods has its own limitations. For example, calculating derivatives or requirement of an initial policy to start off the solution process are some important limitations of the conventional mathematical optimization methods (Mays and Tung 1992). In addition, DP is known to suffer from the "curse of dimensionally" when large scale problems are attempted to solve.

Nowadays, EAs, therefore, have originally been proposed to overcome the limitations of the conventional optimization methods. However, some limitations such as the element of randomness used in generation of initial solutions and generating alternative solutions inherent in most of EAs may reduce their efficiency. In many large scale cases, generally, EAs require additional amount of computer memory which is based on the fact that they need more objective function evaluations than conventional optimization methods. In addition, some EAs such as ACOA have discrete nature and some others have continuous nature. Therefore, ACOA is suitable algorithm for solving discrete problem such as WDS design optimization problem considered here.

ACOA is one of the EAs that has specific characteristic for solving NP-hard combinatorial discrete optimization problems with reasonable computational time cost. For WDS design optimization problem, the pipe diameters are discrete variables in design process and, therefore continuing optimization methods cannot determine them properly. The specific characteristic of ACOA is that in ACOA each artificial ant incrementally builds a solution by adding opportunely defined solution components to a partial solution under construction. This specific unique feature, namely incremental solution building capability, is very useful for solving optimization problems of sequential nature. This algorithm has been inspired in the real ant colony behaviour for finding the shortest path from food source to the nest. Starting with Ant System (AS) (Colorni et al. 1991), a number of algorithmic approaches based on this characteristic of real ant was developed and applied with considerable success to a variety of combinatorial optimization problems from academic as well as from real world applications. Many other algorithms have been proposed to improve the performance of AS, such as Ant Colony System (ACS), Elitist Ant System ( $\left(\mathrm{AS}_{\text {elite }}\right)$, Elitist-Rank Ant System ( $\left.\mathrm{AS}_{\text {rank }}\right)$ and Max-Min Ant System (MMAS). Advancements have been made on the AS to improve the operation of the decision policy and the manner in which the policy incorporates new information to help explore 
the search space of the problem. These developments have primarily been aimed at managing the trade-off between the two contradictory search characteristic of exploration and exploitation. Ali et al. 2009; Ostfeld 2011; Stutzle et al. 2011; and Chandar Mohan and Baskaran 2012) reviewed an amount of research work in the field of using ACOA to solve different optimization problems in the last 20 years. A review of the literature reveals that studies on the application of ACOA to different optimization problems has not been satisfactorily fruitful and is worth further investigation. Numerous papers on the ACOA application for optimization of water engineering problems such as sewer network design (Moeini and Afshar 2013a), WDS design (Zeccchin et al. 2007) and reservoir operation (Moeini and Afshar, 2013b) have been published in recent years.

In this paper, one of the EAs with discrete nature means ACOA is used to effectively solve one of the most important discrete problem means optimal design of WDS by proposing two different methods. In the proposed methods, the ACOA is used to determine pipe diameters as the discrete decision variables of the problem. AS and MMAS referred to ACOA1 and ACOA2 respectively, are applied to determine pipe diameters. MMAS is applied here to overcome the premature convergence problem to suboptimal solution which is well trained in AS by managing the trade-off between the two inconsistent search characteristic of exploration and exploitation. By determining the pipe diameters of WDS, a hydraulic analysis is required. For the hydraulic analysis, in this paper, the ACOA-based models are interfaced with EPANET as simulator. Three benchmark test examples are solved here with proposed methods and the results are presented and compared with those obtained with other existing methods. It is worth noting that many different EAs have been used to solve this problem. However, based on the discrete nature of design of WDS, here, ACOA is used to solve this problem in order to overcome the limitations of pervious works. In some researches, the hydraulic analysis is based on the simplified assumptions such as considering inaccurate value for hydraulic value coefficients equations. However, EPANET simulator is used here for the hydraulic analysis. In addition, in some researches the continues nature of EAs are used to solve discrete nature problem of WDS design and in some other researches the equations of simulation model are solved using iterative numerical or traditional methods and therefore some of the WDS components such as pump cannot be easily modelled. Here, this fact is regarded considering third test example in which the WDS is fed by pump. The efficiency of these innovations is fully presented and highlighted in section "Model application and results" by comparison of the results obtained with proposed methods with other available results.

\section{Ant Colony Optimization Algorithm (ACOA).}

The ACOA is based on the natural foraging behaviour of a colony of real ants to find the shortest path between food source and their nest. When ants are travelling, they deposit a substance called pheromone which is more attractive for other ants to follow them. Ants can smell pheromone and they incline to choose probability paths marked by strong pheromone concentrations when choosing their path. It has been shown experimentally that this pheromone trail following behaviour can give rise to the emergence of the shortest paths once employed by a colony of ants. Based on the real ant behaviour, the ACOA was developed (Colorni et al., 1991). The original and simplest version of ACOA is AS. Later, many other algorithms such as MMAS have been developed to overcome the limitations and improve the performance of the AS. Here, AS and MMAS are used to solve WDS design optimization problem proposing two different methods.

Definition of proper graph is the first step of process for solving an arbitrary optimization problem using ACOA. A graph $G=(D P, O P, C O)$, therefore, can be defined, where $D P=$ $\left\{\mathrm{dp}_{1}, \mathrm{dp}_{2}, \ldots . \mathrm{dp}_{\mathrm{I}}\right\}$ is the set of decision points, $\boldsymbol{O P}=\left\{\mathrm{op}_{\mathrm{ij}}\right\}$ is the set of options $j(j=1,2, \ldots, J)$ at each of the decision point $i(i=1,2, \ldots, I)$ and finally $C O=\left\{\mathrm{co}_{\mathrm{ij}}\right\}$ is the set of costs associated with options $O P=\left\{o p_{i j}\right\}$. (Moeini and Afshar, 2009).

\subsection{Ant System (AS)}

The original and simplest version of ACOA is AS. The basic steps of solving optimization problem using AS can be defined as follows (Colorni et al. 1991): 
1. At the start of the process, some proper values are initialized to the amount of pheromone trail on all options $o p_{i j}$.

2. A colony with the size of $M$ is selected and at one time, each of them is placed randomly on the decision points of the problem.

3. By using a transition rule for selection proper option at each decision point $i$, (Eq. 1), each arbitrary ant, $m$, starts its movement from one decision point to the next and the solutions are incrementally constructed. This procedure is repeated until all decision points of the problem are covered.

$$
p_{i j}(m, t)=\frac{\left[\tau_{i j}(t)\right]^{\alpha}\left[\eta_{i j}\right]^{\beta}}{\sum_{j=1}^{J}\left[\tau_{i j}(t)\right]^{\alpha}\left[\eta_{i j}\right]^{\beta}}
$$

In Eqn. 1, $\mathrm{p}_{\mathrm{ij}}(\mathrm{m}, \mathrm{t})$ is the probability that the ant $m$ selects option $j$ of the $i$ th decision point, $\mathrm{op}_{\mathrm{ij}}$, at iteration $t ; \tau_{\mathrm{ij}}(\mathrm{t})$ is the concentration of pheromone on option $o p_{i j}$ at iteration $t ; \eta_{\mathrm{ij}}$ is the heuristic value of option $o p_{i j}$, and $\alpha$ and $\beta$ are pheromone and heuristic sensitivity parameters, respectively.

4. The cost of the solution, $\mathrm{f}(\varphi)^{\mathrm{m}}$, is calculated when ant $m$ creates a complete solution, $(\varphi)^{\mathrm{m}}$.

5. When steps 3 and 4 are repeated for all ants, $M$, at each iteration $t$, the pheromone is updated using the general form of Eq. 2 for the pheromone updating rule.

$\tau_{\mathrm{ij}}(\mathrm{t}+1)=\rho \tau_{\mathrm{ij}}(\mathrm{t})+\Delta \tau_{\mathrm{ij}}$

In Eqn. 2, $\tau_{\mathrm{ij}}(\mathrm{t}+1)$ is the amount of pheromone trail on option op $\mathrm{ij}_{\mathrm{j}}$ at iteration $t+1$; $\tau_{\mathrm{ij}}(\mathrm{t})$ is the concentration of pheromone on option $\mathrm{op}_{\mathrm{ij}}$ at iteration $t ; \rho(0 \leq \rho \leq 1)$ is the pheromone evaporation coefficient and $\Delta \tau_{i j}$ represents the change in pheromone concentration associated with option $\mathrm{op}_{\mathrm{ij}}$ which is defined as Eqn. 3:

$$
\begin{aligned}
& \Delta \tau_{\mathrm{ij}}=\sum_{\mathrm{m}=1}^{\mathrm{M}} \Delta \tau_{\mathrm{ij}}^{\mathrm{m}}= \\
& \begin{cases}\sum_{\mathrm{m}=1 \mathrm{f}(\varphi)^{\mathrm{m}}}^{\mathrm{M}} & \text { if option jis chosen } \\
0 & \begin{array}{l}
\text { by the best ant } \\
\text { otherwise }
\end{array}\end{cases}
\end{aligned}
$$

In Eqn. 3, $\mathrm{f}(\varphi)^{\mathrm{m}}$ is the cost of the solution produced by the ant $m$ and $R$ is pheromone reward factor.

6. Steps 2 to 5 are continued until convergence criterion is met.

\subsection{Max-Min Ant System (MMAS)}

The most important issue that can be experienced by all ACOAs is premature convergence to suboptimal solutions. To overcome this problem, the MMAS was developed by Stutzle and Hoos (2000). The basis of MMAS is the provision of dynamically evolving bounds on the pheromone trail intensities $\left(\tau_{\mathrm{ij}}(\mathrm{t})\right)$ which are defined as follows (Eqns. 4 and 5):

$$
\begin{aligned}
\tau_{\max }(t) & =\frac{1}{1-\rho} \frac{\mathrm{R}}{\mathrm{f}(\varphi) \text { best }} \\
\tau_{\min }(\mathrm{t}) & =\frac{\tau_{\max }(\mathrm{t})\left(1-\left(\text { p }_{\text {best }}\right)^{1 / \mathrm{I}}\right)}{\mathrm{J}_{\text {avg }} \cdot\left(\text { p }_{\text {best }}\right)^{1 / \mathrm{I}}}
\end{aligned}
$$

In Eqs. 4 and $5, \tau_{\min }(\mathrm{t})$ and $\tau_{\max }(\mathrm{t})$ represent the lower and upper limit on the pheromone trail strength at iteration $t$, respectively; $f(\varphi)^{\text {best }}$ is the cost of the solution produced by the best ant at each iteration, pbest is the probability that the best solution is constructed again; Javg represents the average number of options available at decision points of the problem; and $I$ is the number of decision points.

In MMAS, only the cost of iteration-best ant is used for calculating the change of pheromone 


$$
\begin{aligned}
& \Delta \tau_{\mathrm{ij}}=\Delta \tau_{\mathrm{ij}}^{\text {best }}= \\
& \begin{cases}\frac{\mathrm{R}}{\mathrm{f}(\varphi)^{\text {best }}} & \text { if option } \mathrm{j} \text { is chosen } \\
0 & \begin{array}{l}
\text { by the best ant } \\
\text { otherwise }
\end{array}\end{cases}
\end{aligned}
$$

$\Delta \tau_{\mathrm{ij}}$ which is defined as Eqn. 6.

In Eqn. 6, $\mathrm{f}(\varphi)^{\text {best }}$ is the cost of the solution produced by the best ant. It is worth noting that application of MMAS to some benchmark optimization problems has shown that MMAS overcomes the stagnation problem, hence improves the performance of the ACOAs (Afshar and Moeini 2008).

\section{Water Distribution System Design Optimization Problem}

WDSs consist of interconnected elements such as pipes, tanks, pumps and other components. Such systems are used to transfer treated water from one or more sources to users spread over a wide area. Designing an effective WDS with minimum cost is a quite complex task which can be achieved by formulating and solving it as an optimization problem.

Optimal WDS design requires optimal determination of WDS components such as pipe diameters, tank and pumping station positions and heights leading to a complex optimization problem with a large number of hydraulic constraints. Having a suitable and cost-effective WDS is normally interpreted as finding the solution for this problem that minimizes total cost without violating the constraints.

The standard description form of a WDS design optimization problem with a prespecified layout can be presented as follows. The objective function of this problem can be formulated as Eqn. 7 in which it is minimizing the total construction cost of WDS.

Minimize $\mathrm{C}_{\mathrm{T}}=\sum_{\mathrm{l}=1}^{\mathrm{N}} \mathrm{c}_{\mathrm{l}} \mathrm{L}_{\mathrm{l}}$

In Eqn. 7, $\mathrm{C}_{\mathrm{T}}$ is the total cost of the pipes in the WDS; $L_{l}$ is length of the $l$ th pipe; $c_{l}$ is per unit cost of the $l$ th pipe and $N$ is the total number of existing pipes. The cost function is to be minimized under the following constraints.
Continuity equation: A continuity constraint should be satisfied for each node as follows:

$$
\sum_{\mathrm{l} \in \mathrm{in}(\mathrm{k})} \mathrm{q}_{\mathrm{l}}-\underset{\mathrm{l} \in \operatorname{out}(\mathrm{k})}{\sum \mathrm{ql}_{1}}=\mathrm{Q}_{\mathrm{k}} \quad \forall \mathrm{k}=1, \ldots \ldots \mathrm{K}
$$

In Eqn. 8, $Q_{k}$ is the required demand at consumption node $k ; q_{l}$ is the flow rate in pipe $l$; in $(\mathrm{k})$ is the list of the pipes incoming to node $k$; $\operatorname{out}(k)$ is the list of the pipes out coming from node $k$; and $K$ is the total number of exiting nodes in the WDS.

Energy equation: The energy constraint for each loop in the network of WDS can be written as follows:

$$
\sum_{l \in \mathrm{p}} \mathrm{J}_{1}=0 \quad \forall \mathrm{p}=1, \ldots \ldots \mathrm{P}
$$

In Eqn. 9, $\mathrm{J}_{1}$ is the head loss in the $l$ th pipe and $P$ is the total number of loops in the WDS.

Several equations can be used to evaluate the hydraulic parameters of WDS such as head losses in the pipes. Here, Hazen-Williams equation is used to find hydraulic parameters. Therefore the head losses can be calculated as follows:

$$
\mathrm{J}_{\mathrm{l}}=\mu \mathrm{L}_{\mathrm{l}}\left(\frac{\mathrm{q}_{\mathrm{l}}}{\mathrm{ch}_{\mathrm{l}}}\right)^{\lambda} \mathrm{d}_{\mathrm{l}}^{\gamma} \quad \forall \mathrm{l}=1, \ldots \ldots \mathrm{N}
$$

In Eqn. 10, $\mathrm{J}_{1}$ is the head loss in the $l$ th pipe; $\mathrm{d}_{1}$ is the diameter of pipe $l ; \mathrm{ch}_{1}$ is the HazenWilliams coefficient of pipe $l ; q_{l}$ is the flow rate in pipe $l$ and $\mu, \gamma, \lambda$ are constant coefficients of Hazen-Williams equation.

Nodal pressure head: The maximum and minimum values should be defined for the nodal pressure head of WDS as Eq. 11 in which the nodal pressure head of WDS should be within this range.

$\mathrm{H}_{\text {min }} \leq \mathrm{H}_{\mathrm{K}} \leq \mathrm{H}_{\max } \quad \forall \mathrm{k}=1, \ldots \ldots \mathrm{K}$

In Eqn. 11, $\mathrm{H}_{\mathrm{k}}$ is the pressure head at node $K$; $\mathrm{H}_{\max }$ is the maximum required pressure head; and $H_{\text {min }}$ is the minimum required pressure head.

Flow velocity: The maximum and minimum values should be defined for flow velocity of WDS pipes as Eq. 12 in which the flow velocity of WDS pipes should be within this range. 


$$
\mathrm{V}_{\text {min }} \leq \mathrm{V}_{1} \leq \mathrm{V}_{\text {max }} \quad \forall \mathrm{l}=1, \ldots \ldots \mathrm{N}
$$

In Eqn. 12, $\mathrm{V}_{1}$ is the flow velocity in the $l$ th pipe; $\mathrm{V}_{\max }$ is the maximum flow velocity; and $\mathrm{V}_{\text {min }}$ is the minimum flow velocity.

Commercial pipe diameters: A set of commercially available WDS pipe diameters should be defined for WDS pipe diameters as Eqn. 13 in which the WDS pipe diameters should be selected from it.

$$
\mathrm{d}_{1} \in \boldsymbol{D} \quad \forall \mathrm{l}=1, \ldots, \mathrm{N}
$$

In Eqn. 13, $\mathrm{d}_{1}$ is the diameter of pipe $l$ and $D$ denotes the set of commercially available pipe diameters.

It should be noted that the penalty method is often used to effectively guide solutions of optimization problems from one infeasible solution area to a feasible solution one. Here, the penalty method is applied for formulation of the WDS as an unconstrained optimization problem. In the used method, therefore, nodal pressure head and flow velocity constraints are added in the original objective function. Therefore, a new problem can be defined by the minimization of the penalized objective function of Eq. 14 subject to the constraints defined in Eqns. (8) to (13):

$$
\begin{aligned}
& \mathrm{C}_{\mathrm{p}}=\sum_{\mathrm{i}=1}^{\mathrm{N}} \mathrm{c}_{\mathrm{l}} \mathrm{L}_{\mathrm{l}}+\alpha_{\mathrm{p}}\left(\mathrm{CSV}_{\mathrm{V}}+\mathrm{CSV}_{\mathrm{H}}\right) \\
& \mathrm{CSV}_{\mathrm{V}}=\sum_{\mathrm{l}=1}^{\mathrm{N}}\left(1-\frac{\mathrm{V}_{\mathrm{l}}}{\mathrm{V}_{\min }}\right)^{2}+\sum_{\mathrm{l}=1}^{\mathrm{N}}\left(\frac{\mathrm{V}_{\mathrm{l}}}{\mathrm{V}_{\max }}-1\right)^{2} \\
& \mathrm{CSV}_{\mathrm{H}}=\sum_{\mathrm{k}=1}^{\mathrm{K}}\left(1-\frac{\mathrm{H}_{\mathrm{k}}}{\mathrm{H}_{\min }}\right)^{2}+\sum_{\mathrm{k}=1}^{\mathrm{K}}\left(\frac{\mathrm{H}_{\mathrm{k}}}{\mathrm{H}_{\max }}-1\right)^{2}
\end{aligned}
$$

In Eqn. 14, $\mathrm{C}_{\mathrm{p}}$ is the penalized total cost function of WDS; $\alpha_{p}$ is the penalty parameter with a large enough value to ensure that any infeasible solution will have a higher total cost than any feasible solution; $\mathrm{CSV}_{\mathrm{V}}$ is a measure of the flow velocity constraint violation of the trial solution and $\mathrm{CSV}_{\mathrm{H}}$ is a measure of the nodal pressure head constraint violation of the trial solution. It should be noted that in calculating the CSV, the summation ranges over those nodes and pipes at which a violation of constraints (11) and (12) occurs, that is the terms in parentheses are positive. Other hydraulic constraints are satisfied among using simulation program (EPANET). In other words, EPANET satisfies the continuity and energy conservation equations while calculating the flow rate in each pipe and the pressure head at each node. Furthermore, here, the flow velocity and the nodal pressure head constraint violations have the same penalty parameter value due to the fact that these constrains are normalized.

\section{Methodology}

The WDS design optimization problem is solved here by proposing the simulationoptimization approach. In the proposed approach, the EPANET software is used as a simulator and ACOA is used as an optimization method with the goal of finding pipe diameters. Here, the optimization model is coded in MATLAB and linked with EPANET.

In the proposed approach, at first, a proper graph should be defined to formulate the WDS design as an optimization problem using ACOA. This graph consists of a set of nodes referred to as decision points and edges referred to as options available at each decision points. Here the decision variables of the problem are the pipe diameters of the WDS which are determined in ACOA1 and ACOA2 methods using AS and MMAS, respectively.

In the proposed methods, the pipes of WDS are considered as decision points. The options available at each decision point are, therefore, defined by a finite number of commercially available pipe diameters. Figure 1 represents the problem graph defined for ACOA1 or ACOA2, where vertical lines show the decision points, dashed small lines show the components of commercially available pipe diameters $(j=1, \ldots . . J)$ at each decision point $i(i=1, \ldots . . I)$, and finally the bold small lines show a trial solution on the graph constructed by an arbitrary ant.

By determining the pipe diameters of WDS, the ACOA-based models are interfaced with EPANET for the hydraulic analysis. For both methods, Eqn. 14 is used to calculate the total cost of the trial solutions generated. Finally, optimal pipe diameters are determined using proposed methods. Figures 2 and 3 illustrate the process of solution constructions in ACOA1 and ACOA2 formulations, respectively.

It is worth noting that the different hydraulic softwares have been developed for pipe network analysis such as EPANET, Water CAD, Water GEMS, MIKEURBAN and so on. Each of these software's has its advantages and 
limitations. Due to more advantages of EPANET software, this software is used in this paper. In other words, EPANET is freeware, user friendly and free source software that everybody can simply use it. Due to these noted facts and its accuracy and availability, EPANET is so popular in the field of water distribution analysis and therefore it is used here. EPANET water distribution system simulator is the most commercial hydraulic model which has been developed by the US Environment Protection Agency. The EPANET performs extendedperiod simulation of hydraulic and water quality behaviour within pressurized pipe networks. This well-documented and tested public domain simulator provides a convenient platform for implementing the simulationoptimization approach (Rossman 2000). Here, the EPANET software is linked to MATLAB with the help of EPANET toolkit. EPANET toolkit is a Dynamic Link Library (DLL) of functions and an extension of EPANET simulation package that allows developers to customize EPANET software accordingly (Rossman 1999). If other pipe network analysis software can be linked to MATLAB, these software can be easily considered as simulators.

\section{Model Applications and Results}

In order to show the ability and performance of the proposed methods for the optimal design of WDS, three benchmark examples are applied here with different scales. Therefore, small (test example $I$ ), medium (test example $I I$ ) and large scale (text example III) test examples are presen- ted and solved here using proposed methods. Furthermore, in the third test example, the WDS is fed by a pump; however, in the first and second test examples the WDS is fed by gravity. The first test example $(I)$ as shown in Fig. 4 is the two-loop WDS (Alperovits and Shamir 1977). This WDS network has 7 nodes, 8 pipes and two loops in which it is fed by gravity from a reservoir with a $689(\mathrm{ft})$ fixed head. All WDS network pipes lengths are 3281 (ft) and their Hazen-Williams coefficient are 130. The minimum nodal pressure head is $98.4(\mathrm{ft})$. Fourteen commercial candidate pipe diameters for each pipe with corresponding cost values are listed in Table 1. Details of the network including nodal ground elevations and water demand are given in Table 2.

The second test example (II) is the New York City Water Supply Tunnels (NYCWST) problem. Figure 5 shows the network of this problem in which it consists of 20 nodes connected via 21 pipes (Schaake and Lai 1969). Table 3 represents the pipe lengths, existing diameters, minimum nodal required pressure head and water demands of this network. The original form of this WDS has pressure constraint violations at nodes $16,17,18,19$, and 20. Therefore, this WDS is to be modified by adding duplicate pipes in parallel with the existing pipes to meet the minimum nodal pressure head requirements. For each duplicate pipe, there are 15 different diameter sizes and the option of no pipe as shown in Table 1 with corresponding cost values. This WDS is fed by gravity from a reservoir with a 300 (ft) fixed head. All pipes Hazen-Williams constant are equal to 100 .
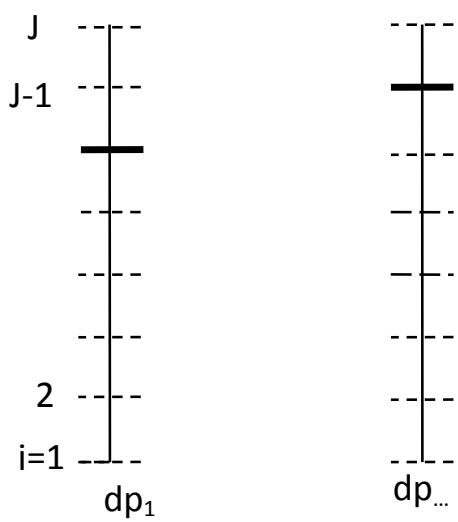

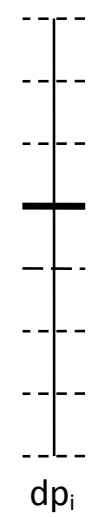

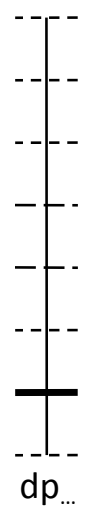

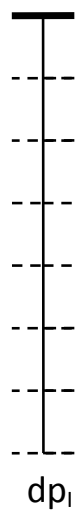

Figure 1. Problem graph defined for ACOA1 or ACOA2. 


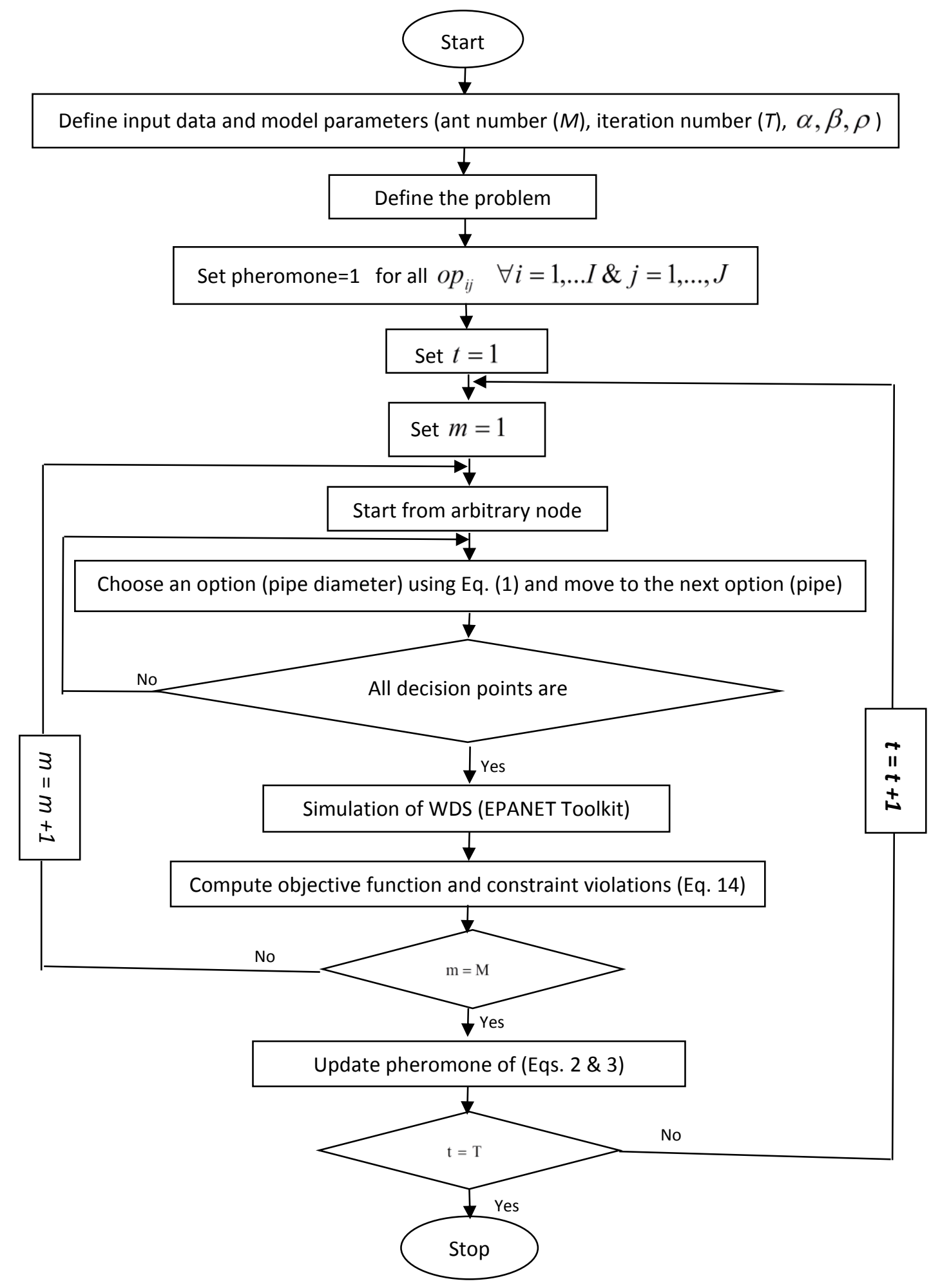

Figure 2. Process of solution constructions in ACOA1. 


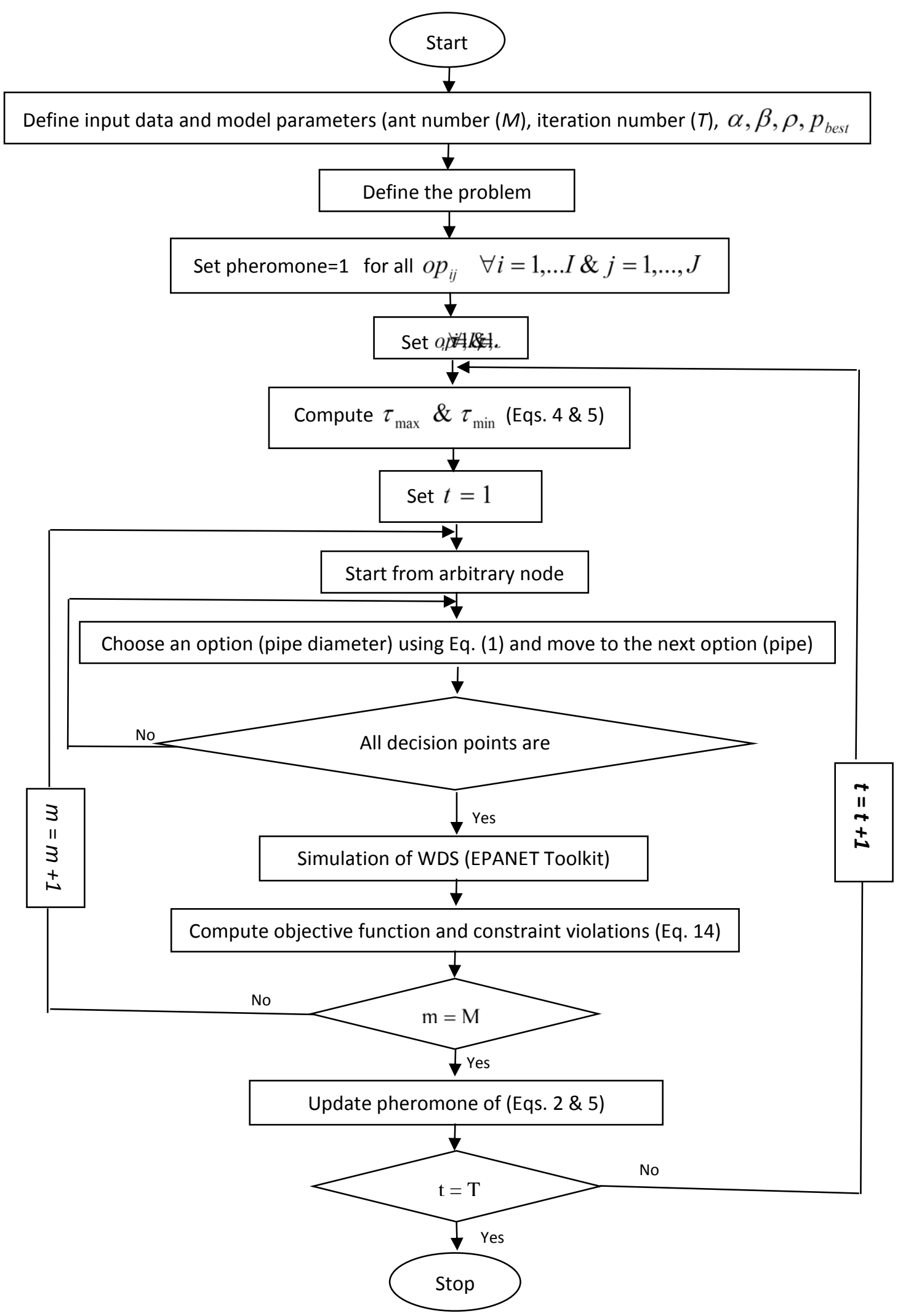

Figure 3. Process of solution constructions in ACOA2. 
Table 1. Candidate pipe diameters and corresponding cost of test examples.

\begin{tabular}{|c|c|c|}
\hline $\begin{array}{c}\text { Test } \\
\text { example }\end{array}$ & Candidate pipe diameters & Cost of candidate pipe diameters \\
\hline I & $\begin{array}{l}\{1,2,3,4,6,8,10,12,14,16,18,20,22,2 \\
4\} \text { (in) }\end{array}$ & $\begin{array}{l}\{2,5,8,11,16,23,32,50,60,90,130,170,300,550\} \\
\text { (units/meter) }\end{array}$ \\
\hline II & $\begin{array}{l}\{36,48,60,72,84,96,108,120,132,14 \\
4,156,168,180,192,204\} \text { (in) }\end{array}$ & $\begin{array}{l}\{93.5,134.0,176.0,221.0,267.0,316.0,365.0,417.0, \\
469.0,522.0,577.0,632.0,689.0,746.0,804.0\} \text { (dollar/ } \\
\text { foot) }\end{array}$ \\
\hline III & $\begin{array}{l}\{80,100,125,150,200,250,300,350\} \\
(\mathrm{mm})\end{array}$ & $\begin{array}{l}\{37890,38933,40563,42554,47624,54125,62109,715 \\
24\} \text { (won/meter) }\end{array}$ \\
\hline
\end{tabular}

Table 2. Nodal data for test example $I$.

Nodal data

\begin{tabular}{c|c|c}
\hline Node & Demand (m3/h) & Ground elevation (m) \\
\hline 1 & Reservoir & 210 \\
2 & 100 & 150 \\
3 & 100 & 160 \\
4 & 120 & 155 \\
5 & 270 & 150 \\
6 & 330 & 165 \\
7 & 200 & 160 \\
\hline
\end{tabular}

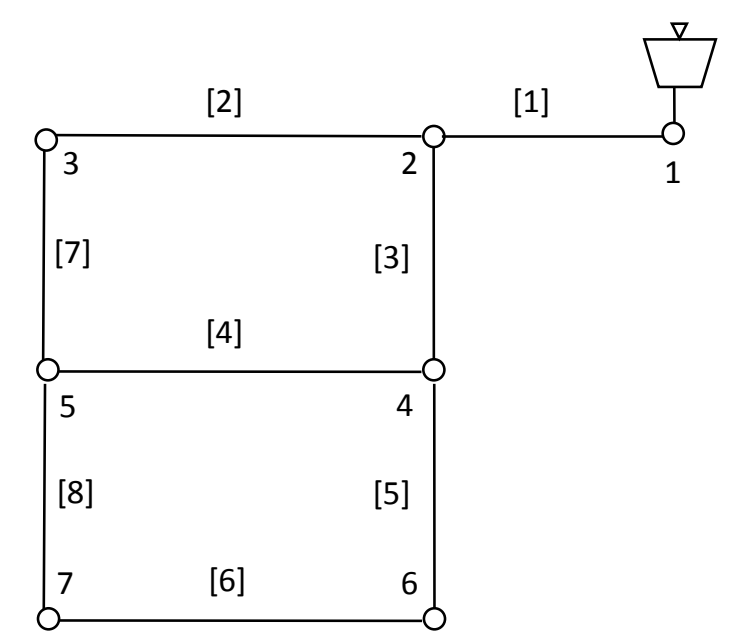

Figure 4. Network of two-loop WDS (test example I). 
Table 3. Pipe and nodal data for test example II.

\begin{tabular}{|c|c|c|c|c|c|c|}
\hline \multicolumn{4}{|c|}{ Pipe data } & \multicolumn{3}{|c|}{ Nodal data } \\
\hline pipe & $\begin{array}{c}\text { Length } \\
(\mathrm{ft})\end{array}$ & $\begin{array}{r}\text { Existi } \\
\text { Diam€ } \\
\text { (in) }\end{array}$ & 51 & Node & $\begin{array}{c}\text { Demand } \\
(\mathrm{Cft} / \mathrm{s})\end{array}$ & $\begin{array}{l}\text { Min } \\
\text { Head } \\
(\mathrm{ft})\end{array}$ \\
\hline 1 & 11600 & 180 & & 1 & Reservoir & 300 \\
\hline 2 & 19800 & 180 & & 2 & 92.4 & 255 \\
\hline 3 & 7300 & 180 & & 3 & 92.4 & 255 \\
\hline 4 & 8300 & 180 & & 4 & 88.2 & 255 \\
\hline 5 & 8600 & 180 & & 5 & 88.2 & 255 \\
\hline 6 & 19100 & 180 & & 6 & 88.2 & 255 \\
\hline 7 & 9600 & 132 & & 7 & 88.2 & 255 \\
\hline 8 & 12500 & 132 & & 8 & 88.2 & 255 \\
\hline 9 & 9600 & 180 & & 9 & 170 & 255 \\
\hline 10 & 11200 & 204 & & 10 & 1 & 255 \\
\hline 11 & 14500 & 204 & & 11 & 170 & 255 \\
\hline 12 & 12200 & 204 & & 12 & 117.1 & 255 \\
\hline 13 & 24100 & 204 & & 13 & 117.1 & 255 \\
\hline 14 & 21100 & 204 & & 14 & 92.4 & 255 \\
\hline 15 & 15500 & 204 & & 15 & 92.4 & 255 \\
\hline 16 & 26400 & 72 & & 16 & 170 & 260 \\
\hline 17 & 31200 & 72 & & 17 & 57.5 & 272.8 \\
\hline 18 & 24000 & 60 & & 18 & 117.1 & 255 \\
\hline 19 & 14400 & 60 & & 19 & 117.1 & 255 \\
\hline 20 & 38400 & 60 & & 20 & 170 & 255 \\
\hline 21 & 26400 & 72 & & & & \\
\hline
\end{tabular}


Table 4. Pipe and nodal data for test example III.

\begin{tabular}{|c|c|c|c|c|}
\hline \multicolumn{2}{|c|}{ Pipe data } & \multicolumn{3}{|c|}{ Nodal data } \\
\hline Pipe & Length (m) & Node & Demand (cmd) & Ground elevation (m) \\
\hline 1 & 165 & 1 & Reservoir & 71 \\
\hline 2 & 124 & 2 & 153 & 56.4 \\
\hline 3 & 118 & 3 & 70.5 & 53.8 \\
\hline 4 & 81 & 4 & 58.5 & 54.9 \\
\hline 5 & 134 & 5 & 75 & 56 \\
\hline 6 & 135 & 6 & 67.5 & 57 \\
\hline 7 & 202 & 7 & 63 & 53.9 \\
\hline 8 & 135 & 8 & 48 & 54.5 \\
\hline 9 & 170 & 9 & 42 & 57.9 \\
\hline 10 & 113 & 10 & 30 & 62.1 \\
\hline 11 & 335 & 11 & 42 & 62.8 \\
\hline 12 & 115 & 12 & 37.5 & 58.6 \\
\hline 13 & 345 & 13 & 37.5 & 59.3 \\
\hline 14 & 114 & 14 & 63 & 59.8 \\
\hline 15 & 103 & 15 & 445.5 & 59.2 \\
\hline 16 & 261 & 16 & 108 & 53.6 \\
\hline 17 & 72 & 17 & 79.5 & 54.8 \\
\hline 18 & 373 & 18 & 55.5 & 55.1 \\
\hline 19 & 98 & 19 & 118.5 & 54.2 \\
\hline 20 & 110 & 20 & 124.5 & 54.5 \\
\hline 21 & 98 & 21 & 31.5 & 62.9 \\
\hline 22 & 246 & 22 & 799.5 & 61.8 \\
\hline 23 & 174 & & & \\
\hline 24 & 102 & & & \\
\hline 25 & 92 & & & \\
\hline 26 & 100 & & & \\
\hline 27 & 130 & & & \\
\hline 28 & 90 & & & \\
\hline 29 & 185 & & & \\
\hline 30 & 90 & & & \\
\hline
\end{tabular}


Table 5. Values of ACOA1 and ACOA2 parameters for all test examples.

\begin{tabular}{lcccccc}
\hline Method & Iteration & Ant & $\alpha$ & $\beta$ & $\rho$ & $p_{\text {best }}$ \\
\hline ACOA1 & 1000 & 100 & 2 & 0.2 & 0.95 & - \\
ACOA2 & 1000 & 100 & 2 & 0.2 & 0.95 & 0.2
\end{tabular}

Table 6. Maximum, minimum and average solution cost values over 10 runs.

\begin{tabular}{|c|c|c|c|c|c|c|c|}
\hline \multirow{2}{*}{$\begin{array}{c}\text { Test } \\
\text { example }\end{array}$} & \multirow[b]{2}{*}{ Method } & \multicolumn{3}{|c|}{ Cost value } & \multirow{2}{*}{$\begin{array}{c}\text { Scaled } \\
\text { Standard } \\
\text { deviation }\end{array}$} & \multirow{2}{*}{$\begin{array}{c}\text { No. of } \\
\text { runs } \\
\text { with } \\
\text { final } \\
\text { feasible } \\
\text { solution }\end{array}$} & \multirow{2}{*}{$\begin{array}{c}\text { CPU } \\
\text { time } \\
\text { for } \\
\text { each } \\
\text { run (s) }\end{array}$} \\
\hline & & Minimum & Maximum & Average & & & \\
\hline \multirow{2}{*}{ I } & ACOA1 & 419000 (units) & 487000 (units) & 433200 (units) & 0.0480 & 10 & 128 \\
\hline & ACOA2 & 419000 (units) & 441000 (units) & 421900 (units) & 0.0163 & 10 & 137 \\
\hline \multirow{2}{*}{ II } & ACOA1 & $71.27(\mathrm{M} \$)$ & $105.98(\mathrm{M} \$)$ & $83.99(\mathrm{M} \$)$ & 0.1188 & 10 & 307 \\
\hline & ACOA2 & $38.64(\mathrm{M} \$)$ & $53.63(\mathrm{M} \$)$ & $45.87(\mathrm{M} \$)$ & 0.1180 & 10 & 327 \\
\hline \multirow{2}{*}{ III } & ACOA1 & 175783163 (Won) & 176018101 (Won) & 175856569 (Won) & 0.0006 & 10 & 367 \\
\hline & ACOA2 & 175783163 (Won) & 175783163 (Won) & 175783163 (Won) & 0 & 10 & 392 \\
\hline
\end{tabular}

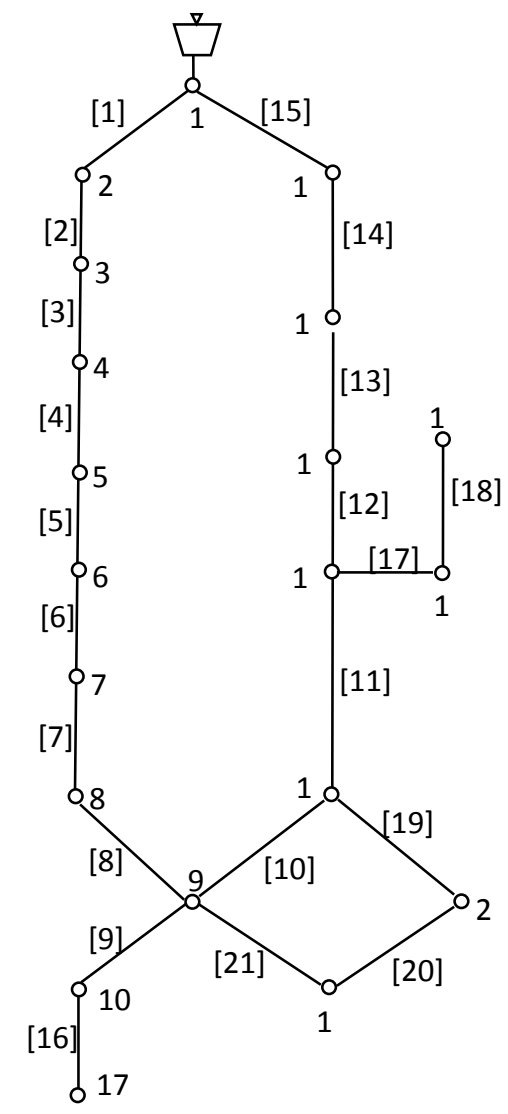

Figure 5. Network of New York WDS (test example II). 
Figure 6 shows the third test example (III) of GoYang network which is first constructed in South Korea (Kim et al. 1994). This WDS network consists of 22 nodes, 30 pipes, 9 loops and is fed by a pump $(4.52 \mathrm{~kW})$ from a reservoir with a 71-m fixed head. Details of the WDS including pipe lengths, and nodal ground elevations and water demands are given in Table 4. The Hazen-Williams constant is equal to 100 for all pipes. The minimum nodal pressure head is 15 meter above ground level. Eight commercial candidate diameters for each pipe with corresponding cost values are listed in Table 1.

A set of preliminary runs is first conducted to find the proper values of AS and MMAS parameters referred to ACOA1 and ACOA2 respectively, as shown in Table 5 for all three test examples. For both methods of all test examples, a colony size of 100 with maximum number of 1000 iterations amounting to maximum number of 100,000 function evaluations is used.

Table 6 shows the results of 10 runs carried out using different randomly generated initial guess for all test examples along with the scaled standard deviation, the number of final feasible solutions and CPU time for each run. It is clearly seen from Table 6 that all measures of the quality of the final solutions such as the minimum, maximum and average cost and the scaled standard deviation are improved when using ACOA2 compared to ACOA1. It should be noted that, due to the fact that premature convergence does not occur in ACOA2 by providing dynamically evolving bounds on the pheromone trail intensities, the ACOA2 has been able to outperform the ACOA1. In other words, the results show the superiority of the MMAS, in conjunction with the ACOA2, over than AS, in conjunction with the ACOA1, in which the trade-off between the two contradictory search characteristic of exploration and exploitation is managed better using MMAS. In order to show the efficiency of proposed methods, these results are compared with those obtained with other existing methods. Comparison of the results shows that the best results are obtained using ACOA2 with no extra computational time cost due to the unique feature of ACOA. Therefore, the proposed method is useful to solve NP-hard combinatorial discrete optimization problems such as optimal design of WDS.

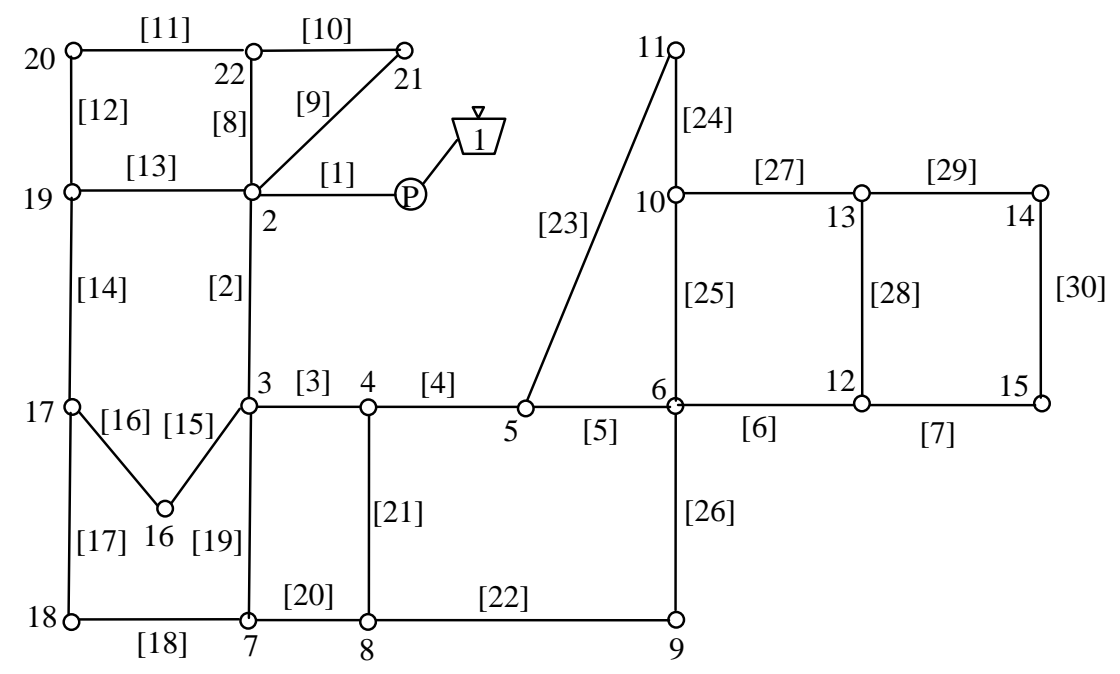

Figure 6. Network of GoYang WDS (test example III). 
Nodal pressure head is one of the most important constraints of WDS design optimization problem. Here, the maximum and minimum pressure head of all test examples obtained with ACOA2 are presented to show the efficiency of proposed methods. For text example $I$, the maximum pressure head occurred at node 2 is $174.72 \mathrm{ft}$ and the minimum pressure head occurred at node 6 is $99.87 \mathrm{ft}$. For text example $I I$, the maximum pressure head occurred at node 2 is $294.207 \mathrm{ft}$ and the minimum pressure head occurred at node 19 is $255.054 \mathrm{ft}$. In addition, in case study II, the pressure head at node 16 and 17 are 260.077 and 272.868 , respectively. Finally, for text example III, the maximum pressure head occurred at node 3 is 27.94 meter and the minimum pressure head occurred at node 15 is 15.1 meter. It is worth noting that the obtained pressure heads are satisfied nodal pressure head for all test examples.

Different methods have been proposed and used to solve text examples I,II and III. At first, the best results obtained with the proposed ACOA2 for test example I are compared with some other available results. The comparison of the results shows that the optimal solution of 419000 units is obtained at the expense of 4700 function evaluations using ACOA2. It is worth noting that the ACOA2 has been able to outperform the methods of Abebe and Solomatine (1999) and Savic and Walters (1997). Furthermore, this compares favourably with about 250000 function evaluations required by the Savic and Walters (1997) using GA1, about 53000 function evaluations required by Cuncha and Sousa (1999) using SA, about 100000 function evaluations required by of Prasad and Park (2004) using GA, about 11155 function evaluations required by of Eusuff and Lansey (2003) using SFLA, about 5100 function evaluations required by of Afshar (2007) using ACO and about 7467 function evaluations required by Wu et al. (2001) using Fast Messy Genetic Algorithm (FMGA) to get the least cost solution of 419000 units. The comparison of these results indicates that the ACOA2 has been able to outperform other methods with no extra computational time cost due to the unique presented feature of this proposed method.

In addition, the results of test example II obtained with the proposed method are compared here with some other available results using different value for constant coefficient of Hazen-Williams equation $(\mu)$. The
ACOA2 is able to get the optimal solution of 38.64 (M\$) in 9900 function evaluations $(\mu=10.669)$. This can be compared approvingly with about 200000 function evaluations required by Murphy et al. (1993) to find the solution of 38.80 (M\$) using GA $(\mu=10.669)$, about 1000000 function evaluations required by Savic and Walters (1997) to find the solutions of $40.42(\mathrm{M} \$)$ and 37.13 (M\$) using GA2 and GA1, respectively, ( $\mu=10.5088)$, about 46016 function evaluations required by Lippai et al. (1999) to find the solution of $38.13(\mathrm{M} \$)$ using TS $(\mu=10.669)$, about 1000000 function evaluations required by Cunha and Sousa (1999) to find the solutions of 37.13 (M\$) using SA $(\mu=10.5088)$, about 37186 function evaluations required by Wu et al. (2001) to find the solutions of $37.83(\mathrm{M} \$$ ) and 37.13 (M\$) using two formulations of Fast Messy Genetic Algorithm named FMGA1 and FMGA2, respectively, $(\mu=10.5088)$, about 31267 function evaluations required by Eusuff and Lansey (2003) to find the solutions of 38.13 (M\$) using SFLA $(\mu=10.669)$, about 6000 function evaluations required by Geem (2006) to find the solutions of $36.66(\mathrm{M} \$)$ using HS $(\mu=10.5088)$, about 7760 function evaluations required by Afshar (2009) to find the solutions of $38.64(\mathrm{M} \$)$ using compact GA (cGA) $(\mu=10.669)$, about 18200 function evaluations required by Afshar (2007) to find the solutions of $38.64 \quad(\mathrm{M} \$)$ using ACO $(\mu=10.669)$, about 13928 function evaluations required by Maier et al. (2003) to find the least-cost solution of $38.64(\mathrm{M} \$)$ using ACOA $(\mu=10.669)$ and finally about 22635 function evaluations required by Zecchin et al. (2006) to find the least-cost solution of 38.64 (M\$) using MMAS $(\mu=10.67)$. It is worth noting that the solutions of Lippai et al. (1999) and Eusuff and Lansey (2003) are infeasible. In other words, the minimum required pressure head is not satisfied at nodes 17 and 19 when obtained diameters are simulated in EPANET software. In addition, a smaller value for constant coefficient of Hazen-Williams equation $(\mu)$ leads to smaller head losses and therefore smaller pipe diameters in which these solutions are infeasible when they are simulated in EPANET software. Comparison of the results shows that ACOA2 has been able to outperform the other used methods with no extra 
computational time cost due to the unique feature of this method which is presented before. In addition, the near optimal solution of Maier et al. (2003), Zecchin et al. (2006) and Afshar (2007), 38.64 (M\$), is obtained here using proposed ACOA2 with smaller computational time.

Finally, the results of test example III obtained with the proposed method are compared with some other available results. The ACOA2 is able to find the optimal solution of 175783163 (Won) in just 8600 function evaluations. This compares favourably with those obtained with other existing methods. Kim et al. (1994) solved the problem using a projected Lagrangian algorithm supported by GAMS/MINOS and then converted the continuous diameters to discrete commercial diameters. They obtained 179142700 (Won) while the original cost was 179428600 (Won). Furthermore, the present result can be compared with about 10000 function evaluations required by Geem (2006) to get the solutions of 177135800 (Won) using HS. It is worth noting that the ACOA2 has been able to outperform all existing methods with no extra computational time cost due to the unique presented feature of this proposed method.

Superior performance of the ACOA2 compared to ACOA1 is already attributed to the provision of dynamically evolving bounds on the pheromone trail intensities and methodology of calculating the change of pheromone $\Delta \tau_{\mathrm{ij}}$. This fact reflected in the typical convergence curves shown in Figs. 7, 8 and 9 for test example I, II and III, respectively. It is seen that the population of ACOA2 has solution costs way below that of ACOA1 due to the fact discussed before.

\section{Conclusion}

In this paper, the Ant Colony Optimization Algorithm (ACOA) was applied to solve Water Distribution System (WDS) design optimization problem using two different methods named ACOA1 and ACOA2. Pipe diameters of the WDS network were determined in both ACOA1 and ACOA2 methods using Ant System (AS) and Max-Min Ant System (MMAS), respectively. For the hydraulic analysis, here, the ACOA-based models were interfaced with EPANET as simulator. Three benchmark test examples were solved using proposed methods and the results were presented and compared.
While two proposed methods showed good performance in solving the problems under consideration, the ACOA2 was observed to produce better results for WDS design optimization problem and to be less sensitive to the randomly generated initial guess required to start the solution process represented by the scaled standard deviation of the solutions produced in ten different runs. In other words, the results showed the superiority of the ACOA2 over other the ACOA1 in which the trade-off between the two contradictory search characteristic of exploration and exploitation was managed better using MMAS which is in conjunction with the ACOA2. It was also shown that the ACOA2 consistently gave better quality solutions than existing traditional and heuristic search methods with less computational effort due to the unique presented feature of this proposed formulation.

\section{Conflict of Interest}

The authors declare no conflicts of interest.

\section{Funding}

No funding was received for this research.

\section{References}

Abebe AJ, Solomatine DP (1999), Application of global optimization to the design of pipe networks. $3^{\text {rd }}$ International Conference on Hydroinformatics, Copenhagen, Balkema, Netherlands, 989-996.

Afshar MH (2007), Application of ant algorithm to pipe network optimization. Iranian Journal of Science and Technology, Transactions B: Engineering 31(B5): 487-500.

Afshar MH (2009), Application of a compact genetic algorithm to pipe network optimization problems. Scientia Iranica. Transactions A: Civil Engineering 16(3): 264271.

Afshar MH, Moeini R (2008), Partially and fully constrained ant algorithms for the optimal solution of large scale reservoir operation problems. Journal Water Resource Management 22(1): 1835-1857.

Ali M, Pant M, Abraham A (2009), A hybrid ant colony differential evolution and its application to water resources problems. In proceedings of the 2009 Nature and 


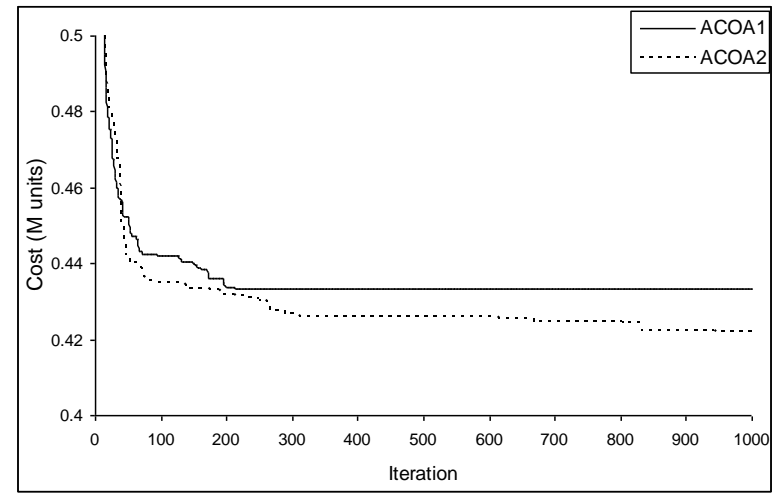

Figure 7. Variation of average solution cost values of test example I using ACOA1 and ACOA2.

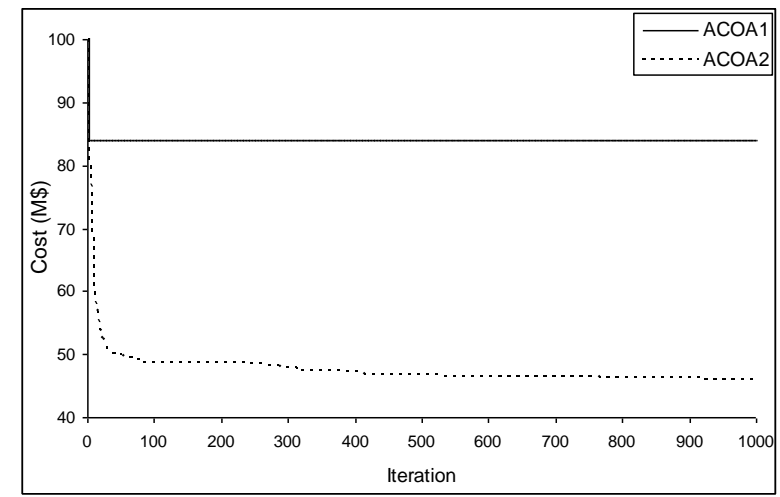

Figure 8. Variation of average solution cost values of test example II using ACOA1 and ACOA2.

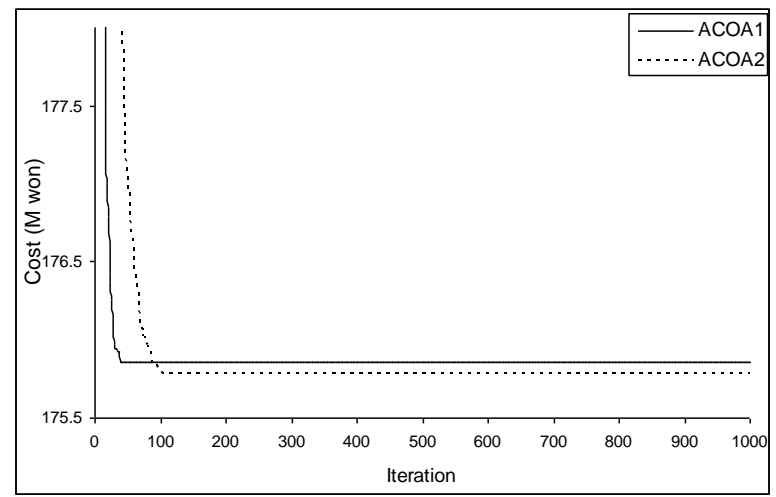

Figure 9. Variation of average solution cost values of test example III using ACOA1 and ACOA2.

Biologically Inspired Computing World Congress $1133-1138$.

Alperovits E, Shamir U (1977), Design of optimal water distribution systems. Water Resources Research 13(6): 885-900.

Babu K, Vijayalakshmi D (2013), Self-adaptive PSO-GA hybrid model for combinatorial water distribution network design. Journal Pipeline System Engineering Practice 4(1): 5767.
Bai D, Yang PJ, Song LX (2007), Optimal design method of looped water distribution network. Systems Engineering-Theory and Practice 27(7): 137-143.

Baoyu Z, Yufei Y, Xin-hua Z (2011), Optimization of water distribution system based on improved shuffled frog leaping algorithm. China Water and Wastewater 27 (9): 45-49. 
Bi W, Dandy GC, Maier HR (2015), Improved genetic algorithm optimization of water distribution system design by incorporating domain knowledge. Environmental Modelling and Software 69: 370-381.

Chandra Mohan B, Baskaran R (2012), A survey: ant colony optimization based recent research and implementation on several engineering domain. Expert Systems with Applications 39: 4618-4627.

Colorni A, Dorigo M, Maniezzo V (1991), Distributed optimization by ant colonies. Proceeding $1^{\text {st }}$ European Conference on Artificial Life (ECAL'91), France, Paris 134142.

Costa ALH, Medeiros JL, Pessoa FLP (2000), Optimization of pipe networks including pumps by simulated annealing. Brazilian Journal of Chemical Engineering, 17 (4-7): 887896.

Cunha MC, Sousa J (1999), Water distribution network design optimization: simulated annealing approach. Journal of Water Resources Planning and Management 125(4): 215-221.

Dong X, Liu S, Tao T, Li SP, Xin KL (2012), A comparative study of differential evolution and genetic algorithms for optimizing the design of water distribution systems. Journal of Zhejiang University SCIENCE A 13(9): 674686.

Eusuff MM, Lansey KE (2003), Optimization of water distribution network design using the shuffled frog leaping algorithm. Journal Water Resource Planning Management 129(3): 210-225.

Geem ZW (2006), Optimal cost design of water distribution networks using harmony search. Engineering Optimization 38(3): 259280.

Gessler J (1985), Pipe network optimization by enumeration. Water Resource Research 23(7): 977-982.

Haghighi A, Samani HMV, Samani ZMV (2011), GA-ILP method for optimization of water distribution networks. Water Resource Management 25(7): 1791-1808.

Jian L, Yanbing J (2010), Optimal design of water distribution system by genetic algorithm. Water and Wastewater Engineering 27(5): 36-39.

Kim JH, Kim TG, Kim JH, Yoon YN (1994), A study on the pipe network system design using non-linear programming. Journal of
Korean Water Resources Association 27(4): 5967.

Lippai I, Heaney JP, Laguna M (1999), Robust water system design with commercial intelligent search optimizers. Journal of Computing in Civil Engineering 13(3): 135143.

Maier HR, Simpson AR, Zecchin AC, Foong WK, Phang KY, Seah HY, Tan CL (2003), Ant colony optimization for design of water distribution systems. Water Resources Planning and Management 129(3): 200-209.

Mays LW, Tung YK (1992), Hydrosystems engineering and management. New York, USA: McGraw-Hill.

Moeini R, Afshar MH (2009), Application of an ant colony optimization algorithm for optimal operation of reservoirs: A comparative study of three proposed formulations. Scientia Iranica. Transactions A: Civil Engineering 16(4): 273-285.

Moeini R, Afshar MH (2013a), Constrained ant colony optimization algorithm for the layout and size optimization of sanitary sewer networks. Urban Water Journal 10(3): 154-173.

Moeini R, Afshar MH (2013b), Extension of the constrained ant colony optimization algorithms for the optimal operation of multi-reservoir systems. journal of Hydroinformatics 15(1): 155-173.

Mohan S, Babu K, (2010), Optimal water distribution network design with honey-bee mating optimization. Journal of Computing in Civil Engineering 24(1): 117-126.

Murphy LJ, Simpson AR, Dandy GC (1993), Design of a network using genetic algorithms. Water 20: 40-42.

Ostfeld A (2011), Ant colony optimization for water resources systems analysis - review and challenges. Ant Colony Optimization Methods and Applications. chapter 17, Published by InTech.

Prasad TD, Park NS (2004), Multiobjective genetic algorithms for design of water distribution networks. Journal of Water Resource Planning and Management 130(1): 73-82.

Rossman LA (1999), The EPANET programmer's toolkit. In proceedings of Water Resources Planning and Management Division Annual Specialty Conference, ASCE, Tempe, AZ, 1-74.

Rossman LA (2000), EPANET 2 users manual. National Risk Management Research Laboratory, office of research and 
development, U.S. EPA, Cincinnati, $\mathrm{OH}$ 45268.

Savic DA, Walters GA (1997), Genetic algorithms for least-cost design of water distribution networks, Journal of Water Resources Planning and Management 123(2): 67-77.

Schaake J, Lai D (1969), Linear programming and dynamic programming - application of water distribution network design. Report 116, MIT Press, Cambridge, MA.

Sheikholeslami R, Zecchin AC, Zheng F,Talatahari S (2016), A hybrid cuckooharmony search algorithm for optimal design of water distribution systems. Journal of Hydroinformatics 18(3): 544-563

Shibua A, Janga Reddya M (2012), Reliabilitybased optimal design of water distribution networks under uncertain demands using cross-entropy method. ISH Journal of Hydraulic Engineering 18(3): 258-268.

Stutzle T, Hoos H (2000), MAX-MIN ant system. Future Generation Computer Systems 16(8): 889-914.

Stutzle T, López-Ibáñez M, Dorigo M (2011), Concise overview of applications of ant colony optimization. Wiley Encyclopedia of Operations Research and Management Science, John Wiley and Sons Inc, 896-911.
Wu ZY, Boulos PF, Orr CH, Ro JJ (2001), Using genetic algorithms to rehabilitate distribution systems. Journal of American Water Works Association 93(11): 74-85.

Xu C, Goulter I (1999), Reliability-based optimal design of water distribution networks. Journal of Water Resource Planning and Management 125(6): 352-362.

Yang L, Sui J, Hua Z (2012), Harmony search algorithm for optimal design of water supply networks. Journal of Theoretical and Applied Information Technology 46(2): 526-536.

Zecchin AC, Simpson AR, Maier HR, Leonard M, Roberts AJ, Berrisford MJ (2006), Application of two ant colony optimization algorithms to water distribution system optimization. Mathematical and Computer Modelling 44: 451-468.

Zecchin AC, Maier HR, Simpson AR, Leonard M, Nixon JB (2007), Ant colony optimization applied to water distribution system design: Comparative study of five algorithms. Journal of water Resources planning and Management 133(1): 87-92.

Zhou X, Gao DY, Simpson AR (2016), Optimal design of water distribution networks by a discrete state transition algorithm. Engineering Optimization 48(4): 603-628. 